\title{
ULTRSONIC BLIND WALKING STICK FOR THE VISUALLY IMPAIRED
}

\author{
Shraddha Bunnan ${ }^{1}$, Gagan Pratap Singh ${ }^{2}$, S.P. Tondare ${ }^{3}$ \\ ${ }^{1}$ BTech Biomedical Engineering, Bharati Vidyapeeth University College of Engineering, Pune \\ ${ }^{2}$ BTech Biomedical Engineering, Bharati Vidyapeeth University College of Engineering, Pune \\ ${ }^{3}$ Assistant Professor, Department of Electronics, Bharati Vidyapeeth University College of Engineering, Pune
}

\begin{abstract}
The man kind has been suffering with many diseases and abnormalities. Blindness is one of the disabilities which is very common and difficult to treat. The main motive of this paper is to help the blind people with a good and easy to use blind walking stick that uses a microcontroller to detect obstacles in front of him with many additional features which are quite cost effective. The stick will be meant for the rehabilitation of the blind people. The product is based on the distance measurement property of the ultrasonic sensors. It will measure the distance and velocity of the obstacle in the way of the person and will alarm the person about any obstacle (moving/stationary). The stick will allow detection of obstacles on the ground, holes and pits, uneven surfaces, steps, and other typical obstacles in the path of the person. Since the device is incorporated with wireless module it will be very handy and will be much easier to use as compared to the similar products available in the market. The white cane or stick is mostly preferred by the blind people for walking. But the limitation to the white cane is that the information obtained by the blind user is only by touching the objects by the tip of the cane, which is not completely reliable. Through this proposed paper we offer to give a solution which overcomes the limitations of the ordinary white cane. The ultrasonic blind walking stick is fully automated, comfortable to use and cheap. In other words, it is quite economic over the conventional method.
\end{abstract}

Keywords: Ultrasonic, Blind Walking Stick, Blindness,

\section{INTRODUCTION}

BLINDNESS is caused basically due to the lack of vision. It is the loss of vision which cannot be corrected with glasses or contact lenses. According to WHO (World Health Organisation) 285 million people are estimated to be visually impaired worldwide, 39 million are blind and 246 have low vision.

About $90 \%$ of the world's visually impaired live in lowincome settings. $82 \%$ of people living with blindness are aged 50 and above. An estimated 19 million children are visually impaired.

The white cane or stick is mostly preferred by the blind people for walking. But the limitation to the white cane is that the information obtained by the blind user is only by touching the objects by the tip of the cane, which is not completely reliable.

Through this proposed paper we offer to give a solution which overcomes the limitations of the ordinary white cane. The ultrasonic blind walking stick is fully automated, comfortable to use and cheap. In other words, it is quite economic over the conventional method.

\section{EXISTING METHODS}

There are many new different innovations brought about in the Ultrasonic Blind walking stick.

- One of them is the Voice based Blind walking stick which makes use of programmed voice for guidance.

- Another application makes use of Android technology for navigation purpose.

- There are walking sticks available with GPS system installed in it.

The impact of such applications helps a lot to the blind or visually impaired people in making their life easy. But for starters it could be too confusing for the blind people to use it. It could confuse the blind people for understanding the voice or the gps guiding system. This could cause discomfort or reluctance is using the new technology stick. Also such advanced sticks are very expensive. This is where the proposed stick could be fruitful. 


\section{METHODOLOGY}

\subsection{System Configuration of Proposed Method}

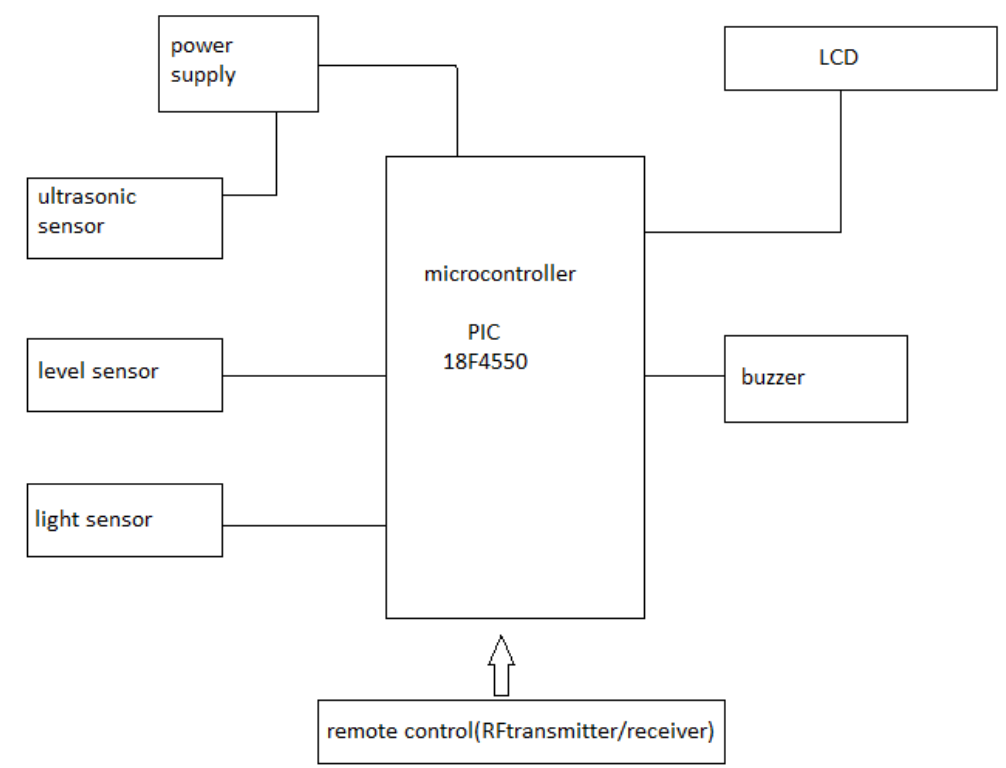

Fig 3.1: Block diagram of the ultrasonic blind walking stick

This blind walking stick allows the visually challenged people to navigate with ease using today's technology. The stick is integrated with an ultrasonic sensor along with water and light sensor (which in this case we use a level sensor and LDR circuit). The ultrasonic sensors are used to detect the obstacles ahead with the help of the ultrasonic waves which further passes the data to the microcontroller. The microcontroller we used is PIC18F4550. The microcontroller further processes the data and calculates the distance if the obstacle. It sendsthe signal to the buzzer with gives out an alarm. It also detects and buzzes a different alarm if the stick enters water and alerts the blind. One more additional feature is that the blind is alerted with one more different buzzer if he enters a light or a dark room. This system also has a intriguing feature to help the blind user to find their stick if they forget or loss it. A normal tv remote control is used for this purpose. Thus the stick provides with obstacle detection along with finding the stick if it gets misplaced

\section{CIRCUIT DIAGRAM:}

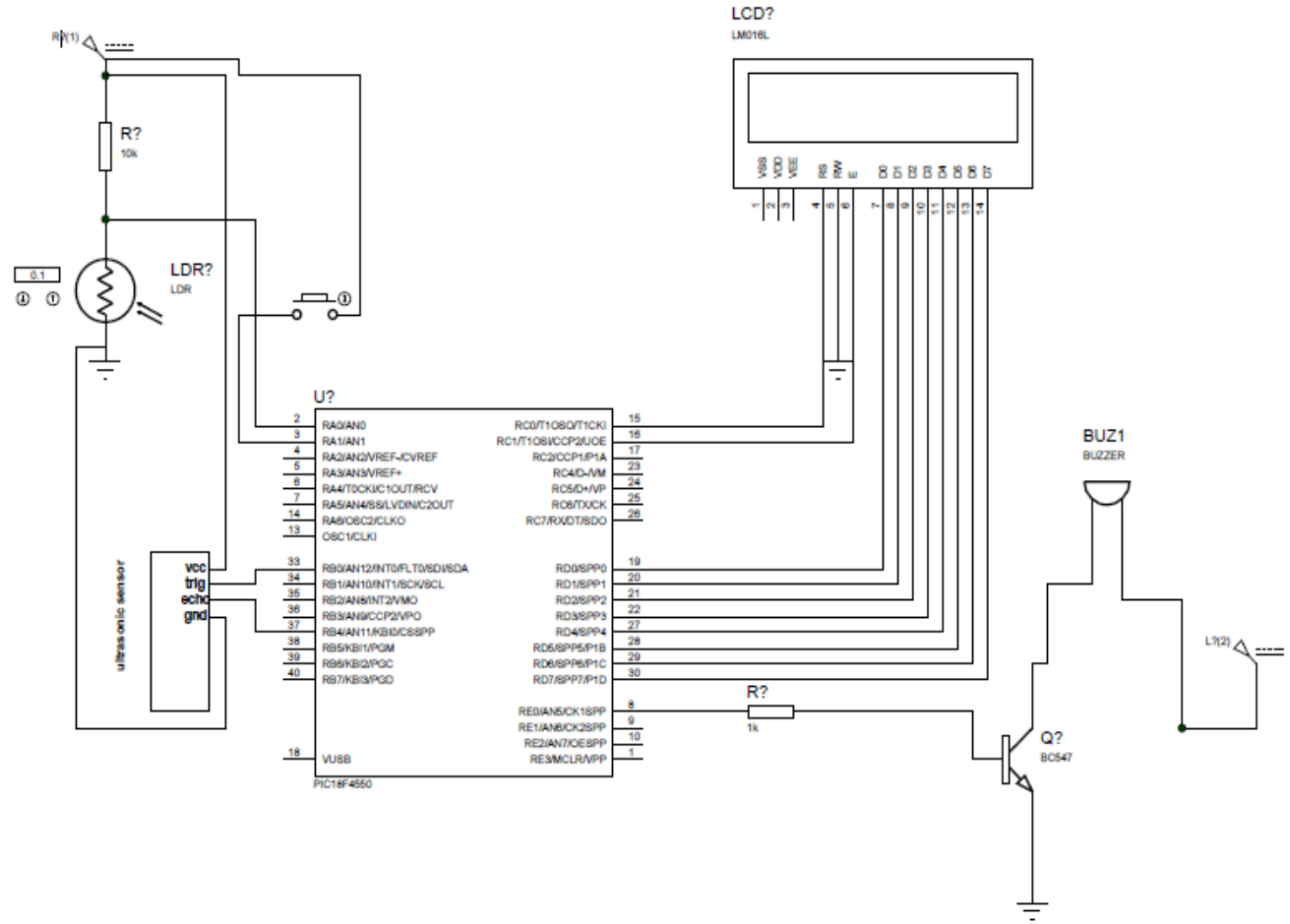

Fig 3.2: Circuit diagram of the Ultrasonic blind walking stick 


\section{RESULTS AND CONCLUSION}

\subsection{Working With Subject}

\section{STEPS:}

1. Power supply is given to the stick through batteries.

2. Obstacle is introduced in front of the stick.

3. Stick is immersed in water.

4. Stick is kept in the dark room.

5. Remote control is used to detect the stick which is kept at a certain distance.

\section{RESULTS}

1. The obstacle is detected by the stick and user is notified by a buzzing alarm.

2. Level sensor detects the water and notifies the user with a different buzzing sound.

3. The LDR detects the darkness in the room and notifies the user with another buzzing sound.

4. Again fourth variation of the buzzer sound is used to locate the stick whenever you press the button on remote.

\section{CONCLUSION}

The blind walking stick has the following features:

Entirely automated. 2. Can be maintained \& operated easily. 3.Very comfy to function. 4.Authentic \& Durable. 5. Low power consumption. 6.sThe Microcontroller can be code protected. 7. Simplicity of the design makes it effective navigation assistant .7 . Wet or muddy or potentially slippery terrain can be detected by a pair of electrodes. 8. Overall manufacturing cost is low \& parts are available in both local \& international market.

\section{REFERENCES}

[1]. Bousbia-Salah, M., M. Bettayeb, and A. Larbi, “A navigation aid forblind people", Journal of Intelligent \& Robotic Systems, 2011. 64(3-4): p. 387-400.

[2]. Pradeep, V., G. Medioni, and J. Weiland."Robot vision for thevisually impaired", in Computer Vision and Pattern Recognition Workshops (CVPRW), Computer Society Conference on. 2010. IEEE.

[3]. Faria, J., et al. "Electronic white cane for blind people navigation assistance", in World AutomationCongress (WAC), 2010. IEEE.

[4]. Nunokawa, K., S. Ino, and K. Doi, "Vibration of the White Cane Causing a Hardness Sense of an Object", in HCIInternational 2013-Posters' Extended Abstracts. 2013, Springer. p. 493-497.

[5]. Hersh, M., "Deafblind people,stigma and the use of communication and mobility assistive devices",Technology and Disability, 2013. 25(4): p. 245-261. 